\title{
Three-dimensional architecture of the ligamentum teres in the human hip joint
}

\author{
Nobuhiro Kaku ${ }^{1}$ \\ Tatsuo Shimada ${ }^{2}$ \\ Tomonori Tabata ${ }^{1}$ \\ Hiroaki Tagomori ${ }^{1}$ \\ Tetsutaro Abe ${ }^{1}$ \\ Juan Juan Zhang ${ }^{3}$ \\ Hiroshi Tsumura ${ }^{1}$
}

1 Department of Orthopaedic Surgery, Faculty of Medicine, Oita University, Yufu City, Oita, Japan

2 Oita College of Judo Therapy and Acupuncture-

Moxibustion, Yufu City, Oita, Japan

3 Department of Matrix Medicine, Faculty of Medicine, Oita University, Yufu City, Oita, Japan

\section{Corresponding Author:}

\section{Nobuhiro Kaku}

Department of Orthopaedic Surgery,

Faculty of Medicine, Oita University

1-1 Idaigaoka Hazama-machi

879-5593 Yufu City, Oita, Japan

E-mail: nobuhiro@oita-u.ac.jp

\section{Summary}

Background: We aimed to investigate the threedimensional structure of the collagenous fibers of the ligamentum teres (LT) of the human hip and clarify the LT micro-anatomy at the attachment of the femoral head.

Methods: Femoral heads and LT were collected during hip arthroplasty. Specimens were cut into 5-10-mm squares, prepared, developed, and observed under a light microscope. Next, specimens were prepared and examined under a scanning electron microscope (SEM).

Results: Under optical microscope, LT adhered to the artificial cartilage at the attachment of the femoral head. Under SEM, LT comprised parallelly arranged collagenous fibers and the fine collagenous fibrils were twisted. While the central collagenous fibers of the LT at the attachment of the femoral head penetrated the articular cartilage tissue and reached the ring-shaped bone, fibers at the margin traversed and adhered to the cartilage surface.
Conclusion: Articular cartilage and subchondral bone are present at the LT attachment to the femoral head. Although collagenous fibers of the LT show parallel arrangement at the main trunk, they are dispersed at the cartilage surface and not all reach the thin subchondral bone of the femoral head. This could possibly weaken ligament strength at the attachment of the femoral head.

Level of evidence: IV.

KEY WORDS: collagenous fibers, ligamentum teres, light microscope, scanning electron microscope.

\section{Background}

The human intra-articular ligaments include the ligamentum teres (LT) of the hip, and the anterior cruciate ligament and posterior cruciate ligament of the knee joint. Because the ligaments of the knee joint contribute to joint stability, their damage leads to joint instability and consequently the development of osteoarthritis ${ }^{1}$. On the other hand, it has been also reported that some injuries of the LT, including femoroacetabular impingement and acetabular dysplasia, are a cause of osteoarthritis in the hip joint 2,3 . However, because there are no detailed descriptions in these reports, it is still unclear whether the LT is ruptured as a consequence of the anatomical elements of the bone or the instability of the hip joint. Thus, it is also not clear whether the cause of osteoarthritis is the anatomy of the hip or a secondary consequence of LT rupture, or whether the cartilage injury is the consequence of the micro-instability. Recently, clinical attention has been paid to the LT and the possible involvement of the ligament in hip joint stability and in disease progression ${ }^{4,5}$. The LT is known as an important stabilizer of the hip joint, particularly during hip flexion and external rotation ${ }^{5}$. Though older age and acetabular bony pathomorphology are two of the known risk factors for LT tears ${ }^{5}$, we are interested in whether the LT itself has a microanatomical vulnerable feature. The LT in the human joint starts from the fovea of the femoral head and involves a ligament attached to the acetabular notch. Macroscopically, it appears to be attached to the articular cartilage of the femoral head. However, there is doubt as to whether this ligament truly adheres to the articular cartilage. If this is true, this contradicts the definition of a liga- 
ment. Fundamental elucidation of the anatomy and functionality of the LT is yet to be reported. In particular, there is no detailed report on the micro-anatomy of the LT. Details of the morphology and the arrangement of collagenous fibers, which are a main component of LT attachment and the ligament itself, have not been fully elucidated. Furthermore, no reports have ultrastructurally examined the LT using an electron microscope. The purpose of this study was to clarify the characteristics of the micro-anatomy of the $\mathrm{LT}$, especially its construction, the arrangement of the collagenous fibers contained within the LT, and the structure of the region where the ligament adheres to the femoral head, by using an optical microscope [light microscope (LM)] and scanning electron microscope (SEM). Though older age and acetabular bony pathomorphology are two of known risk factors for LT tears ${ }^{6}$, we are interested in whether the LT itself has micro-anatomical vulnerable feature.

\section{Materials and methods}

Femoral heads removed at the time of arthroplasty for femoral neck fractures were used. The femoral heads and LT were collected during surgery from a 76-year-old woman, a 79-year-old woman, and an 80year-old man without any history of disease at the time of surgery for the femoral neck fracture. The femoral heads were placed immediately in formalin fixative and cut with a diamond band saw longitudinally to allow adequate view of the LT. The cut samples were immersed in 5\% EDTA solution for 2 weeks and demineralized. For the LM, 7- $\mu \mathrm{m}$ paraffin sections were stained with hematoxylin and eosin ( $\mathrm{HE}$; $\mathrm{pH} 2.5)$ and Alcian blue $(\mathrm{pH} 1.0)$ for histochemical carbohydrate reactions. For the SEM, after observation with an optical microscope, the remaining paraffin blocks were deparaffinized with xylene and refixed with a mixed solution of glutaraldehyde and formaldehyde. Furthermore, samples were immersed in $2 \mathrm{~N}$ $\mathrm{NaOH}$ solution at $37^{\circ} \mathrm{C}$ for 3 hours to digest the extracellular matrix. Samples were thoroughly washed with physiological saline and placed in $1 \%$ osmium tetroxide, $1 \%$ tannic acid, and $1 \%$ osmium tetroxide for 1 hour each, dehydrated with alcohol, and then freeze-dried with butyl alcohol. After evaporation, they were examined using the S-4800 SEM (Hitachi, Tokyo, Japan $)^{7}$.

\section{Light microscopic examinations}

Specimens were cut into 5-10-mm squares, fixed in $10 \%$ formalin or $4 \%$ paraformaldehyde in phosphate buffer ( $\mathrm{pH} 7.4$ ), dehydrated, and embedded in paraffin. The 7- $\mu \mathrm{m}$ sections were stained with HE. They were also immunostained with rabbit anti-desmin antibody (DAKO Corporation, Glostrup, Denmark), immersed in rabbit IgG gold solution (5 nm) (Amersham Pharmacia Biotech, Little Chalfont, UK), physically developed ${ }^{7,8}$, and observed under a LM (Nikon, Tokyo, Japan).

\section{Electron microscopic examinations}

For immunohistochemistry, small tissue blocks including Purkinje cells from a sheep heart were fixed in periodate-lysine-paraformaldehyde fixative, dehydrated, and embedded in London resin (LR white). Ultrathin sections were immunostained using the IgG gold-silver method with anti-desmin antibody, stained with only uranyl acetate, and examined with a JEOL 100CX electron microscope (JEOL USA, MA, USA).

To visualize intermediate filaments by SEM, the sheep false tendon fixed in one-quarter Karnovsky fixative was immersed in $40 \%$ dimethyl sulfoxide solution, freeze-dried, and treated with $0.3 \%$ saponin solution for 3 hours at $37{ }^{\circ} \mathrm{C}^{9}$. However, the remaining paraffin blocks of human hearts used for LM were also used for SEM. They were deparaffinized with $x y$ lene, hydrated, fixed again in Karnovsky fixative, and immersed in $2 \mathrm{~N} \mathrm{NaOH}$ at $37{ }^{\circ} \mathrm{C}$ for 3 hours to expose myofibrils and intermediate filaments ${ }^{7}$. All specimens were placed in $1 \%$ osmium tetroxide, $1 \%$ tannic acid, and $1 \%$ osmium tetroxide for 1 hour each, dehydrated in ethanol of ascending concentrations, and dried with t-butyl alcohol using a freeze-drying method. Specimens were coated with gold and examined at 25 or $15 \mathrm{kV}$ using an HFS-II or Hitachi S-800 SEM (Hitachi, Tokyo, Japan).

The procedures in this study followed were performed ethically in accordance with international standards and as required by Muscles, Ligaments and Tendons Journal, as described in Padulo et al. ${ }^{10}$. This research was approved by the Ethics Committee of our University. The approval number and approval date were 1053 and 22 July 2016, respectively. All experimental procedures were performed in accordance with the guidelines for the care and use of laboratory animals of Animal Experimentation of our University.

\section{Results}

\section{Macroscopic and light microscopic findings}

Macroscopically, the LT was attached to the fovea of the femoral head, which was in zone 6 of division according to Ilizaliturri et al. ${ }^{11}$ (Fig. 1A). Slightly whitened articular cartilage could be identified on the surface of the femoral head after cleaving the femoral head at the center of the LT with a diamond saw (Fig. 1B, 1C, 1D). The LT spread like a fan in the proximity of the attachment and appeared to adhere to the cartilage. The cartilage width of the ligament attachment appeared to be thinning. When viewing the demineralized specimen under the optical microscope and using hematoxylin and eosin (HE) staining, the LT was enclosed in the ligament membrane and consisted of close, parallel, collagenous fibers. The ligament seemed to adhere to the articular cartilage, but this we were not certain (Fig. 2A, B). Articular cartilage stained with Alcian blue at the site of LT adherence appeared spotted and cartilage tissue appeared sparse (Fig. 2C, 2D). Articular cartilage showed morphological features similar to hyaline cartilage. 

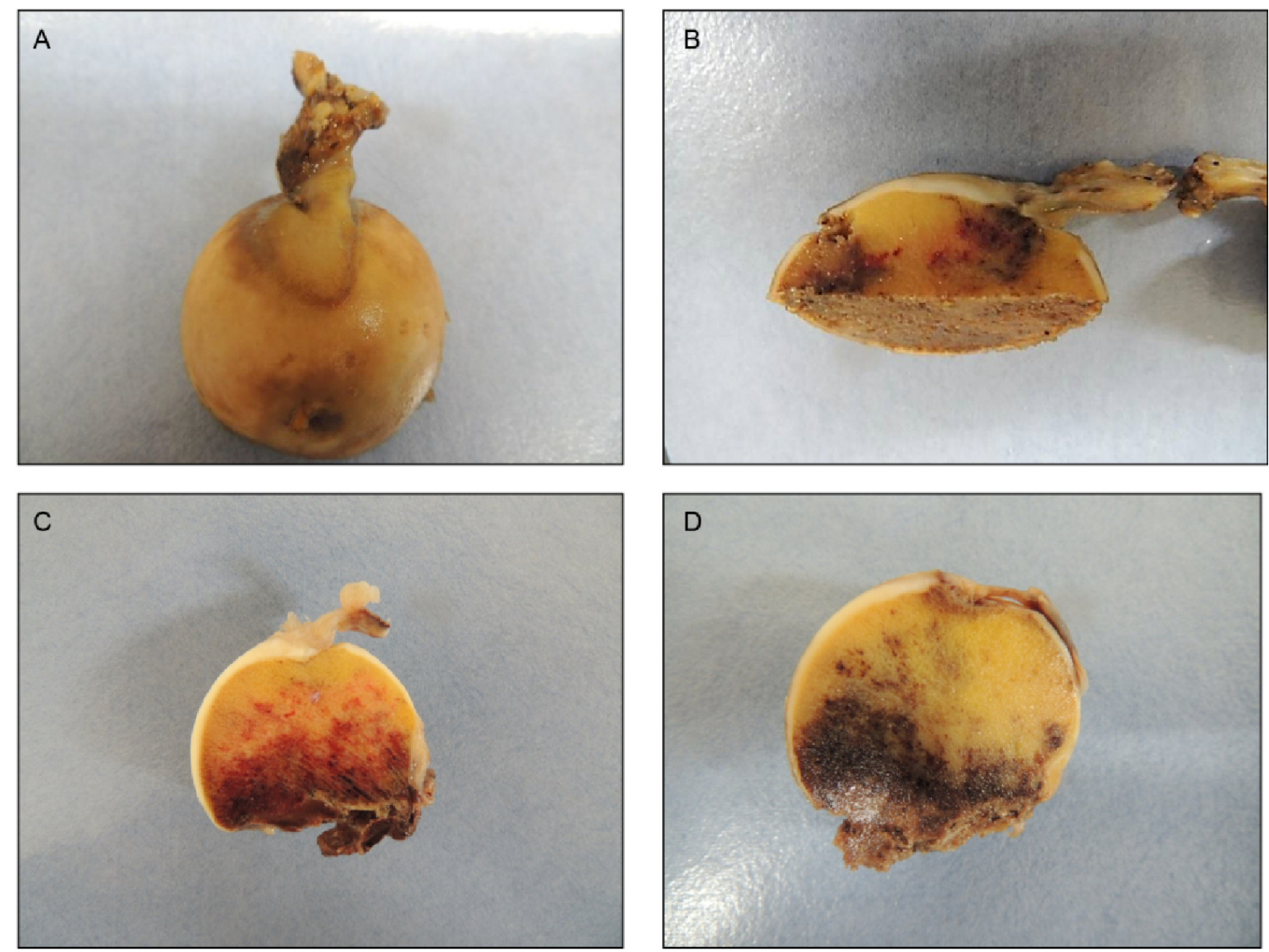

Figure 1. Naked eye view of the femoral head. The LT seems to adhere to zone 6 of division according to Ilizaliturri et al. ${ }^{11}$ of the femoral head (A). When the femoral head is cleaved at the center of the LT with a diamond saw, articular cartilage is identified on the surface (B-D). The LT spreads like a fan near the attachment (C). The cartilage width of the ligament attachment appears thin $(B, D)$.

\section{Scanning electron microscopic findings}

In this study, $2 \mathrm{~N} \mathrm{NaOH}$ treatment with deparaffinized block digested the extracellular matrix and made it possible to three-dimensionally observe collagenous fibers comprising ligaments, cartilage, and bone with the SEM. The string-like ligament spread in a fan shape along the cartilage in the femoral head attachment (Fig. 3). The ligament comprised large and small collagenous fibers arranged in parallel, with each consisting of a bundle of type I collagen fibrils with further twisting viewed in the magnified image (Fig. 4A, 4B). The collagen fibril diameter was 120$140 \mathrm{~nm}$. Ligament, articular cartilage, bone, and bone marrow were identified from the surface in the SEM image of the ligament attachment (Fig. 5). The terminal gradually became thinner and appeared to adhere to the surface of articular cartilage. Collagen fibrils of the ligament appeared to run linearly. However, the collagenous fibrils of the articular cartilage were slightly narrow in diameter and had a network arrangement of approximately 80-100 nm. Fibers at the margin of the ligament traversed the cartilage surface, but when the central part of the ligament adhered to the articular cartilage, the collagenous fibers descended perpendicularly to the femoral head. Branching of the collagen fibers appeared to penetrate the articular cartilage tissue (Fig. 6). In an enlarged SEM image, it was shown that articular cartilage, comprising chondrocytes and collagenous fibrils, was arranged densely with a surrounding network (Fig. 7). The collagenous fibril bundle of the branched ligament traveled linearly within the cartilage tissue (hyaline cartilage) (Fig. 8). Collagenous fibers of the ligament branch that invaded the cartilage reached the ring-shaped bone (Fig. 9). It is noteworthy that the annual ring of the bone comprised thin fiber bundles in which the collagenous tissue fibers were twisted and arranged in a mixed weave (Fig. 10).

\section{Discussion}

A ligament comprises strong connective tissue that connects bone to bone. The anterior cruciate ligament and the posterior cruciate ligament of the knee, which are intra-articular ligaments similar to the $\mathrm{LT}$, have been proven macroscopically and histologically ${ }^{12,13}$ to 

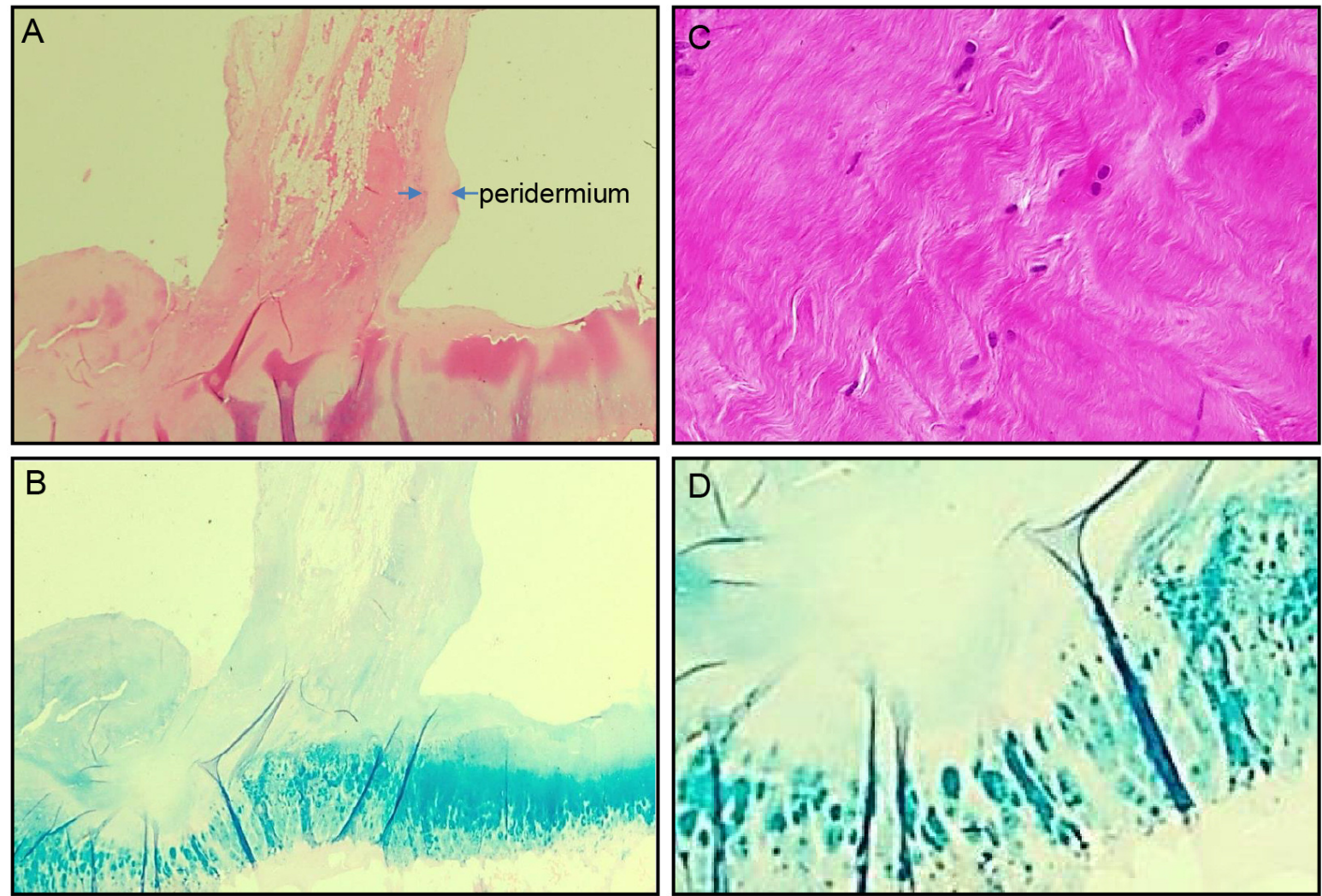

Figure 2. Optical microscopic photograph of the LT attachment area. Using hematoxylin and eosin (HE) staining, the peridermium, which is a dense connective tissue consisting of crossing collagen fibers, is seen surrounding the LT, and the LT appears to adhere to articular cartilage (A). The ligament comprises dense, parallel-arranged, collagenous fibers (B). Articular cartilage is stained with Alcian blue (C). The site where ligaments adhere to the articular cartilage shows sparse cartilage tissue (D).

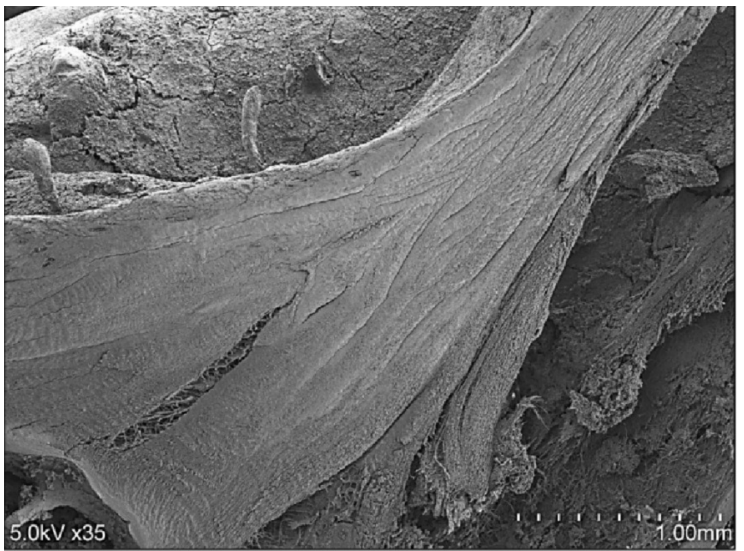

Figure 3. Scanning electron microscope (SEM) image of the longitudinally-cut LT. The string-like femoral head (tough ligament) spreads like a fan at the femoral head attachment.

have fibrous tissue that reaches the bone. It is reported that the LT also starts in the acetabular notch and transverse ligament and ends at the fovea of the femoral head with two or three fiber bundles from the pubis and ischium of the acetabular fossa ${ }^{14,15}$

The anterior bundle that connects with the pubic part of the acetabular notch and the posterior bundle that connects with the ischiate part appear strong, and the gap between the anterior bundle and the posterior bundle is slightly thin. The LT has a thickness of approximately $30-35 \mathrm{~mm}$ and its cross-section has a circular or elliptical fibrous structure ${ }^{16}$. Blood vessels nourishing the femoral head pass during early childhood. In adults, the branches of the closed artery are running, but one-third are retracted ${ }^{17}$. Sensory receptors in the femoral head ligament exist for innervation 8,18 .

Although the function of the LT as a stabilizer within the normal range of motion other than dislocation is largely unknown, it has been clarified, using arthroscopy and MRI, that the LT works to stabilize the femoral head, in particular during flexion, adduction, and external rotation ${ }^{14,16}$. It has actually been reported that damage to the LT, acetabular labrum, and cartilage are observed at the same time. It has been suggested that damage to the LT possibly leads to microinstability of the hip joint ${ }^{19}$. In addition, control of exercise by sensory receptors, blood supply to the femoral head, and agitation of synovial fluid have 

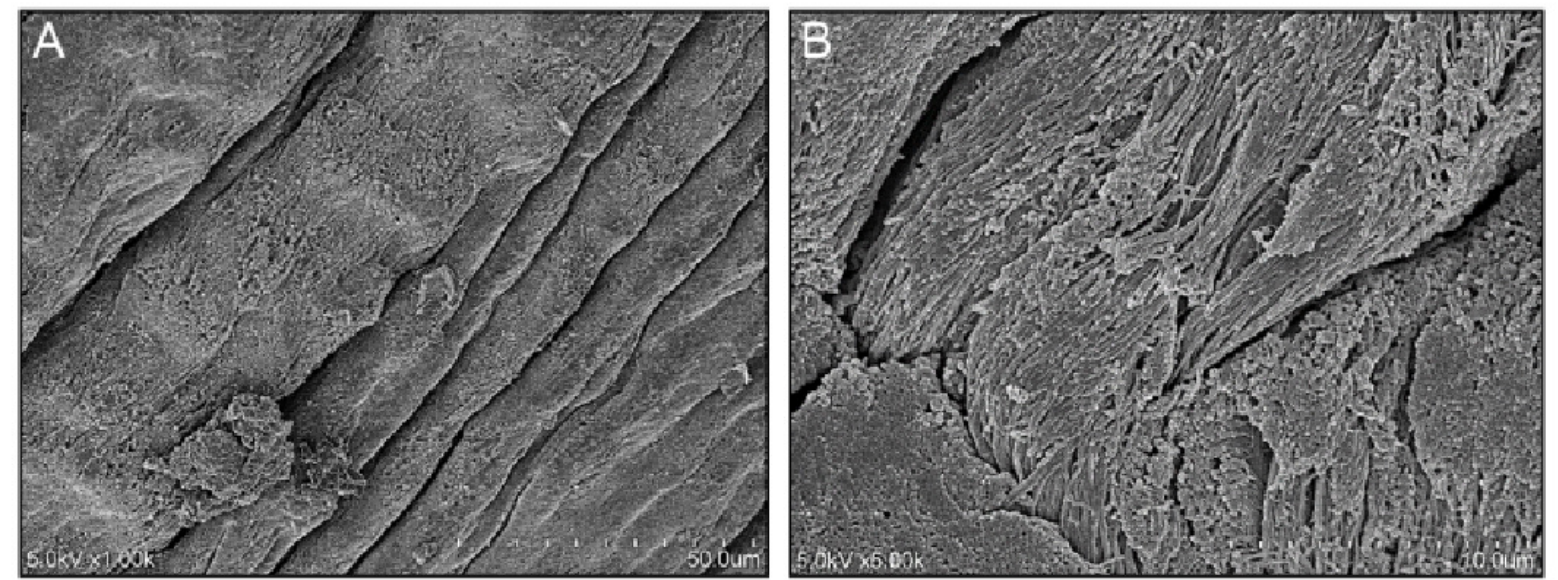

Figure 4 A, B. An enlarged scanning electron microscope (SEM) image of the LT. The ligament consists of large and small, parallel-arranged, collagenous fibers $(A)$. In the enlarged image of the collagen fiber, the collagen fibrils show a torsional arrangement $(\mathrm{B})$.

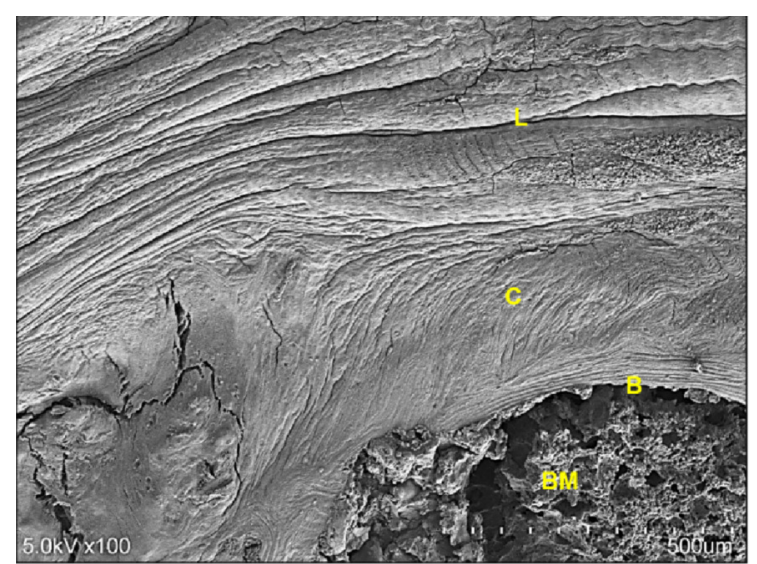

Figure 5. Scanning electron microscope (SEM) image of the ligament attachment area. The bone ligament attachment consists of the ligament (L), articular cartilage $(C)$, bone $(\mathrm{B})$, and bone marrow $(\mathrm{BM})$ from the surface.

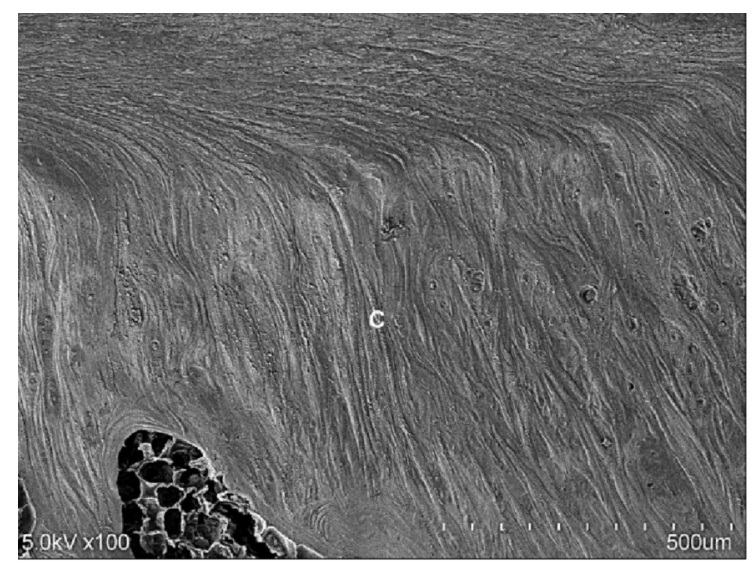

Figure 6. Scanning electron microscope (SEM) image inside the articular cartilage. The collagenous fibers traveling along the cartilage surface appear to dig into the deep layer of the cartilage tissue $(\mathrm{C})$ while changing the angle.

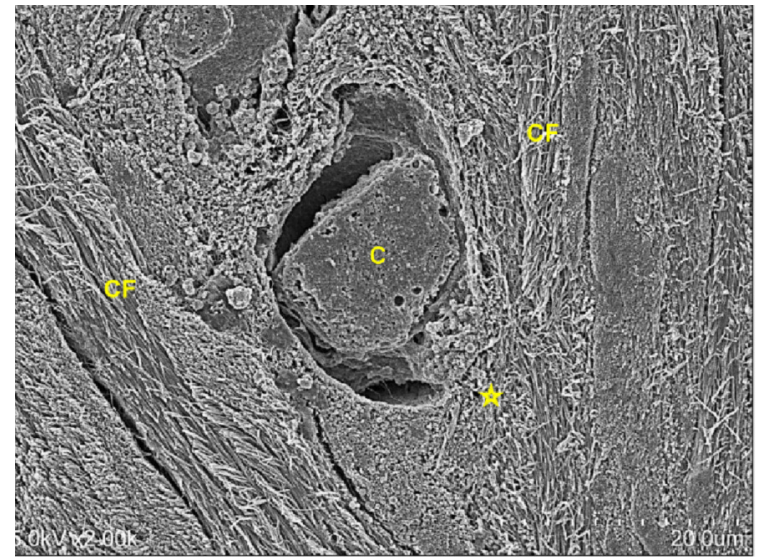

Figure 7. Enlarged image of the inside of the articular cartilage. The collagenous fibril bundle (CF) of the branched ligament travels linearly within the cartilage tissue (hyaline cartilage). C: chondrocytes; *: type II collagen.

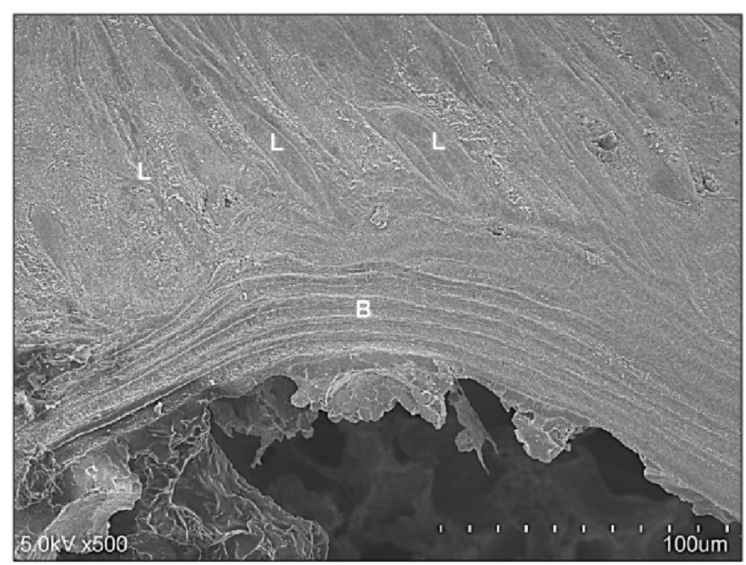

Figure 8 . Boundary between articular cartilage and bone denseness. Ligament branches $(\mathrm{L})$ and collagen fibers that have invaded the cartilage reach the annual ring-shaped bone tissue (B). 


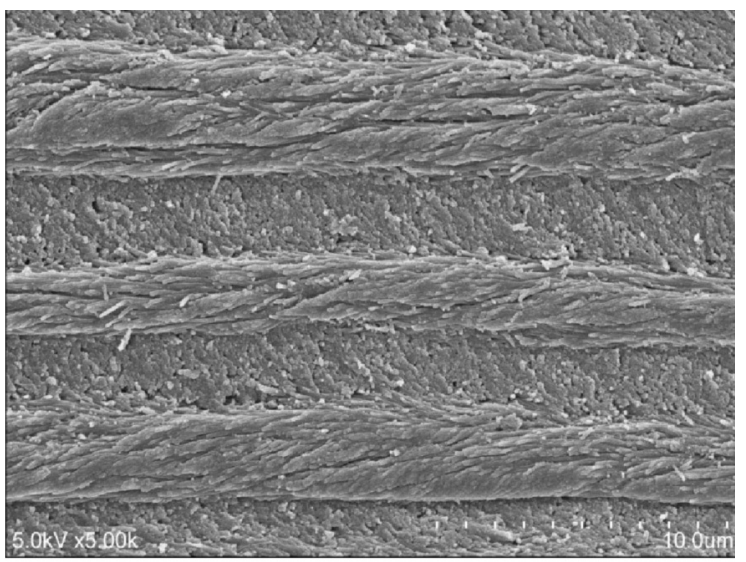

Figure 9. Collagen fibers comprising densities. Twisted collateral fibrils are arranged in a mixed weave. and adhered to the bone. Therefore, our findings support the definition of a ligament. The collagen fibers spread like a fan when reaching the cartilage, and the fibers in the central part of the ligament directly entered the cartilage and reached the bone. As collateral fibers traverse the cartilage surface and move into the cartilage, the collagen fibers straighten to the bone so that the collagen fibers become angled at the surface of the cartilage. Furthermore, collateral fibers at the margin traversed to the cartilage surface, gradually thinned, and stopped on the cartilage surface. In this way, the microanatomical characteristics of the LT, whereby the collagenous fibers dispersed and bent at an angle at the surface of the cartilage and not all collagenous fibers adhered to the bone, may lead to a reduction of ligament strength at the attachment of femoral head. In addition, though it was diffi-

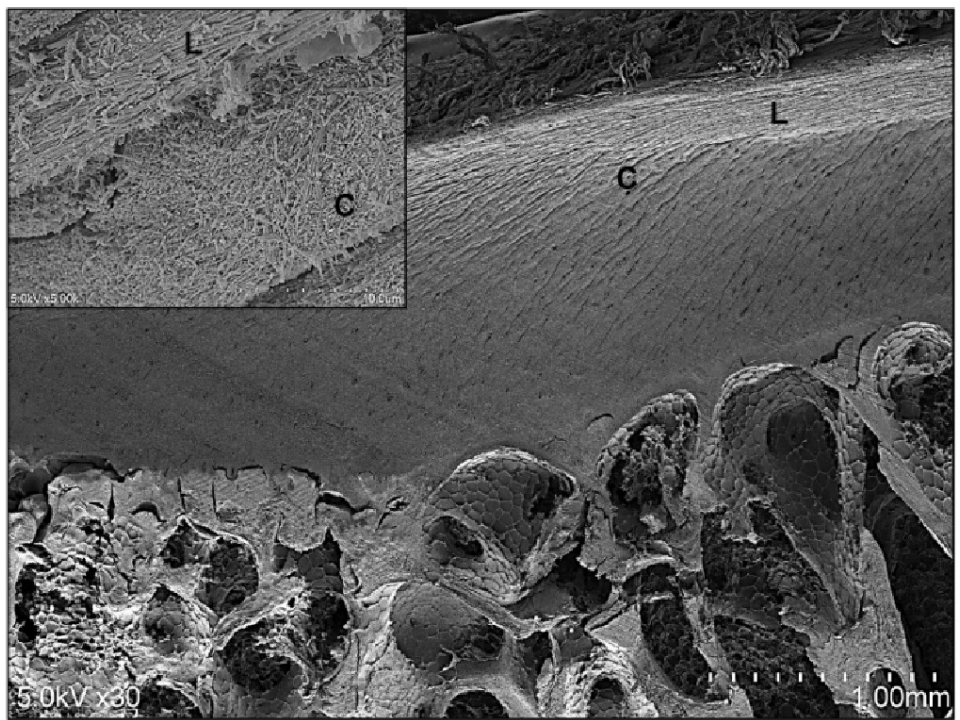

Figure 10. Margin of the ligament attachment. Collagen fibers ( $L$ ) of ligaments become thinner as they go to the periphery. Finally, they appear to adhere to the cartilage surface $(\mathrm{C})$. Insertion shows collagenous fibrils $(\mathrm{L})$ comprising ligaments and collagenous fibrils $(\mathrm{C})$ comprising cartilage.

been reported as LT functions ${ }^{16}$.

However, the structure and function of the femoral head ligament have not yet been clarified. In fact, it has been reported that there is no cartilage in the fovea of the femoral head attachment ${ }^{16,19}$. However, this SEM study utilizing the chemical digestion method revealed that the collagenous fibers which formed the fiber bundle at the middle of the ligament branched to each fiber at the time of reaching the cartilage of the femoral head. Furthermore, individual fibers (although not all fibers) reached the subchondral bone linearly after passing the cartilage independently.

Collagenous fibrils composed of type II collagen in the articular cartilage were thinner than those of the ligaments, so it was relatively easy to distinguish them from each other. Therefore, although the LT appeared macroscopically to be attached to the cartilage surface, collagenous fibers in the central part of the ligament actually passed through the cartilage cult to confirm macroscopically, this study revealed that articular cartilage and subchondral bone certainly exists at the fovea of the femoral head. More remarkable was the thickness of the subchondral bone. Because our specimens were from femoral neck fractures of elderly patients, it cannot be denied that osteoporosis was involved. However, our specimens were very thin, approximately $30-50 \mu \mathrm{m}$, in this series.

As a limitation of this study, we did not consider the details of the ligament attachment on the acetabulum side. This is required to clarify the difference between the acetabular side and the adhesion form. Clinically, it may not be necessary to collect acetabular bones with ligaments. Collection from fresh cadavers is one option.

Finally, we conclude that the LT in the human hip joint consists of large and small collagenous fibers arranged in parallel. At the femoral head attachment, the ligament spreads like a fan along the cartilage 
surface. Each collagenous fiber consists of a bundle of collagen fibrils with further twisting. Collagen fibers at the margins of the ligament traverse the cartilage surface and the central collagenous fibers of the ligament descend perpendicularly to the femoral head. Collagenous branches enter the articular cartilage tissue and reach the thin cortical bone of the femoral head. This study was able to elucidate the microanatomy of the collagen fibers of the ligament trunk at the attachment to the femoral head fossa. We believe that our findings help to clarify the involvement of the LT in stability and disease of the hip in more detail. Further investigations of the microanatomy of the attachment at the acetabular fossa of the LT, and the differences in micro-anatomy of the LT caused by diseases and age, are needed in the future.

\section{Conflict of interest}

The Authors have no financial or personal relationships with other people or organizations that could inappropriately influence their work.

\section{References}

1. Louboutin H, Debarge R, Richou J, et al. Osteoarthritis in patients with anterior cruciate ligament rupture: a review of risk factors. Knee. 2009;16:239-244.

2. Kaya M, Suziki T, Minowa T, Yamashita T. Ligamentum teres injury is associated with the articular damage pattern in patients with femoroacetabular impingement. Arthroscopy. 2014;30:1582-1587.

3. Li T, Zhang M, Wang H, Wang Y. Absence of Ligamentum Teres in Developmental Dysplasia of the Hip. J Pediatr Orthop. 2015;35:708-711.

4. Kraeutler MJ, Garabekyan T, Pascual-Garrido C, Mei-Dan O. Hip instability: a review of hip dysplasia and other contributing factors. Muscles Ligaments Tendons J. 2016;21:343-353.

5. Bolia I, Chahla J, Locks R, Briggs K, Philippon MJ. Microinstability of the hip: a previously unrecognized pathology.Muscles Ligaments Tendons J. 2016;21:354-360.
6. Kraeutler MJ, Garabekyan T, Pascual-Garrido C, Mei-Dan O. Ligamentum teres tendinopathy and tears. Muscles Ligaments Tendons J. 2016;21:337-342.

7. Yoshimura A, Yamaguchi T, Kawazato H, Takahashi N, Shimada T. Immuno-histochemistry and three-dimensional architecture of the intermediate filaments in Purkinje cells in mammalian hearts. Med Mol Morphol. 2014;47:233-239.

8. Gerhardt M, Johnson K, Atkinson R, et al. Characterisation and classification of the neural anatomy in the human hip joint. Hip Int. 2012;22:75-81.

9. Sugi Y. Visualization and identification of cytoskeletal filaments in embryonic chick heart by the quick-freeze deep-etch method combined with immunocytochemistry. J Electron Microsc (Tokyo). 1989;38:126-131.

10. Padulo J, Oliva F, Frizziero A, Maffulli N. Muscles, Ligaments and Tendons Journal - Basic principles and recommendations in clinical and field science research: 2016 update. MLTJ. 2016;6(1):1-5.

11. Ilizaliturri VM Jr, Byrd JW, Sampson TG, et al. A geographic zone method to describe intra-articular pathology in hip arthroscopy: cadaveric study and preliminary report. Arthroscopy. 2008:24:534-539.

12. Hatsushika D, Nimura A, Mochizuki T, Yamaguchi K, Muneta T, Akita K. Attachments of separate small bundles of human posterior cruciate ligament: an anatomic study. Knee Surg Sports Traumatol Arthrosc. 2013;21:998-1004.

13. Helito CP, Demange MK, Bonadio MB, et al. Anatomy and Histology of the Knee Anterolateral Ligament. Orthop J Sports Med. 2013;1:2325967113513546.

14. Martin HD, Hatem MA, Kivlan BR, Martin RL. Function of the ligamentum teres in limiting hip rotation: a cadaveric study. Arthroscopy. 2014;30:1085-1091.

15. Bardakos NV, Villar RN. The ligamentum teres of the adult hip. J Bone Joint Surg Br. 2009;91:8-15.

16. Cerezal L, Kassarjian A, Canga A, et al. Anatomy, biomechanics, imaging, and management of ligamentum teres injuries. Radiographics. 2010;30:1637-1651.

17. Chen HH, Li AF, Li KC, Wu JJ, Chen TS, Lee MC. Adaptations of ligamentum teres in ischemic necrosis of human femoral head. Clin Orthop Relat Res. 1996;328:268-275.

18. Sarban S, Baba F, Kocabey Y, Cengiz M, Isikan UE. Free nerve endings and morphological features of the ligamentum capitis femoris in developmental dysplasia of the hip. J Pediatr Orthop B. 2007;16:351-356.

19. Cerezal L, Arnaiz J, Canga A, et al. Emerging topics on the hip: ligamentum teres and hip microinstability. Eur J Radiol. 2012;81:3745-3754. 